Original Article

\title{
Factors Associated with Low Admission Platelet Count in Adults with Acute Aortic Dissection
}

\author{
Shuwen Li, MD, ${ }^{1}$ Jiakai Lu, MD, ${ }^{1}$ Weiping Cheng, MD, ${ }^{1}$ Junming Zhu, MD, ${ }^{2}$ \\ and $\mathrm{Mu} \mathrm{Jin}, \mathrm{MD}^{3}$
}

\begin{abstract}
Purpose: Platelets are crucial components of the coagulation processes, and low admission platelet count (PLC) is associated with adverse clinical outcomes in patients with Stanford type $A$ acute aortic dissection (AAD).

Methods: A total of 130 consecutive patients undergoing Stanford type A AAD surgery in Beijing Anzhen Hospital were enrolled between January 2013 and July 2014. Preoperative clinical and laboratory data from patients were collected. Multiple regression analyses were used to determine the independent factors of low admission platelets.

Results: Adjusted multiple regression analysis showed that age $(\beta:-1.069,95 \%$ confidence interval $[\mathrm{CI}]:-\mathbf{2 . 1 0 9},-\mathbf{0 . 0 2 9})$, $\operatorname{sex}(\beta:-29.973,95 \%$ CI: $-56.512,-3.433)$, tissue factor pathway inhibitor (TFPI; $\beta: 0.197,95 \%$ CI: $0.039,0.354$ ), fibrinogen degradation product (FDP) $(\beta:-0.476,95 \%$ CI: $-0.879,-0.074)$, and attack time ( $\beta: 11.125,95 \%$ CI: 7.963 , 14.287) were significantly associated with admission PLC. Admission PLC increased with attack time up to the 3 days ( $\beta$ : 16.2, 95\% CI: 12.1, 20.2).

Conclusions: We found that increasing age, male patients, patients with lower serum levels of TFPI and higher serum levels of FDP, and patients with a shorter attack time were significantly associated with lower PLC at admission. Moreover, the turning point of attack time is $\mathbf{3}$ days after the onset of dissection.
\end{abstract}

Keywords: platelet count, acute aortic dissection, multiple regression analysis, admission

${ }^{1}$ Department of Anaesthesiology, Beijing AnZhen Hospital, Capital Medical University, Beijing, China

${ }^{2}$ Department of Cardiology surgery, Beijing AnZhen Hospital, Capital Medical University, Beijing, China

${ }^{3}$ Department of Anaesthesiology, Beijing Friendship Hospital, Capital Medical University, Beijing, China

Received: August 6, 2018; Accepted: October 22, 2018

Corresponding author: Mu Jin, MD. Department of Anesthesiology, Beijing Friendship Hospital, Capital Medical University, 95 Yongan, Xicheng, Beijing 100050, China

Email: jinmu0119@hotmail.com

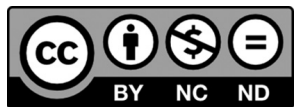

This work is licensed under a Creative Commons Attribution-NonCommercialNoDerivatives International License.

(C)2019 The Editorial Committee of Annals of Thoracic and Cardiovascular Surgery

\section{Introduction}

Stanford type A acute aortic dissection (AAD) represents a serious cardiovascular disease with a high mortality and morbidity. ${ }^{1-4)}$ The period of 14-day after onset has been designated the acute phase, with a mortality rate of $1 \%-2 \%$ per hour early after symptom onset.5) Blood flow through the false lumen is a powerful activator of platelets and the coagulation/fibrinolytic system. Decreased platelet function and platelet count (PLC) have been observed in patients with type A AAD. ${ }^{6,7)}$ It has been proposed that a reduction in PLC might correlate with the excessive consumption of platelets in response to thrombosis of the false lumen during AAD. Moreover, platelet activation in AAD can activate 
attached leukocytes and induce further activation of endothelial cells by releasing pro-inflammatory mediators in response to more severe ischemia-reperfusion injury and systemic inflammation. ${ }^{8-10)}$ Therefore, it is reasonable to speculate that the reduced PLCs are associated with adverse clinical outcomes. ${ }^{11)}$ Recently, accumulating evidence has demonstrated that PLC is positively associated with survival. ${ }^{7,12}$ However, the factors related to PLC upon admission have not been clarified in patients with type A AAD. Thus, the aim of this study was to evaluate the factors related to low admission PLC in patients with type A AAD.

\section{Methods}

\section{Study design and population}

This study was a retrospective analysis of prospectively collected data, which resulted from a previous clinical trial (ClinicalTrials.gov, Identifier: NCT01894334) and full details of the methods have been previously published ${ }^{13)}$ and were approved by the Beijing Anzhen Hospital Clinical Research Ethics Committee (Identifier: 2012013). Patients with Stanford type A AAD were eligible if they were 18- to 75-years old and were suitable for emergency surgery. A total of 130 consecutive patients undergoing Stanford type A AAD surgery in Beijing Anzhen Hospital were enrolled between January 2013 and July 2014. All of the patients enrolled in this study had normal hepatic and renal function. Exclusion criteria included patients with coronary heart disease, heart failure, severe cardiac tamponade, unstable hemodynamics, nervous system abnormalities, and clinically apparent malperfusion. ${ }^{14)}$ In brief, all cases were class Aa according to the Penn Classification, ${ }^{15)}$ which is the absence of branch vessel malperfusion or circulatory collapse. Patients prescribed non-steroidal anti-inflammatory drugs or corticosteroids before or after admission were also excluded. ${ }^{13)}$

\section{Data collection}

The investigators and the participants were not informed of the results during the study. The data were collected through assessments using the data collection protocol. ${ }^{13)}$ In brief, demographics and etiology, such as Body Mass Index (BMI), regular use of prescription drugs, smoking history, hypertension history, and diabetes history, were recorded at recruitment. The onset time of sudden severe chest or upper back pain, loss of consciousness, shortness of breath, sudden difficulty speaking, loss of vision, and weakness or paralysis of one side of the body were recorded. The attack time was defined as the time between disease onset and urgent surgery. At admission, hemodynamic performance, echocardiographic data, and EuroSCORE values were recorded. Venous blood samples were drawn, centrifuged, and stored at $-70^{\circ} \mathrm{C}$ until subsequent use. Plasma levels of interleukin-6 (IL-6), IL-10, tissue factor (TF), tissue factor pathway inhibitor (TFPI), human leukocyte elastase (HLE), tumor necrosis factor alpha (TNF $\alpha)$, vascular endothelial growth factor (VEGF), prostaglandin $\mathrm{I}_{2}\left(\mathrm{PGI}_{2}\right)$, and thromboxane $\mathrm{B}_{2}$ $\left(\mathrm{TXB}_{2}\right)$ were assayed by enzyme-linked immune absorbent assay according to the manufacturer's instructions (Multiskan MK3 Automatic microplate reader, Thermo Fisher Scientific, Waltham, MA, USA). Methane dicarboxylic aldehyde (MDA), myeloperoxidase (MPO), total anti-oxidation capacity (TAOC), and total superoxide dismutase (TSOD) were evaluated by ultravioletvisible spectroscopy. PLC, hemoglobin ( $\mathrm{Hb}$ ), white blood cell (WBC), lactic acid (LAC), fibrinogen (FIB), and fibrinogen degradation product (FDP) were determined by standard quantitative assay techniques in the hospital's Clinical Laboratory Center.

\section{Patient and public involvement}

Patients were not involved in the development of the research question, the design, recruitment, or conduct of this study. Patients who participated did so anonymously, and therefore the study team will be unable to disseminate the results to study participants.

\section{Outcomes}

The primary outcomes were independent factors correlating with admission PLC in patients with type A AAD. Additionally, variation in preferences for demographics and etiology characteristics (e.g., gender, age), hemodynamic performance (e.g., blood pressure, heart rate), and serological results at admission (e.g., IL-6, TFPI, TNF $\alpha$, and $\mathrm{TXB}_{2}$ ) were also assessed.

\section{Statistical analysis}

All data analyses were performed with EmpowerStats software (www.empowerstats.com, X\&Y solutions, Inc. Boston, MA, USA) and R software (http://www.R-project. org). Quantitative variables were presented as the mean \pm standard deviation or median (interquartile range [IQR]), and categorical variables were presented as frequencies or percentages. Univariate and multiple regression analyses were used to determine the independent factors of admission PLC. In univariate analysis, variables associated 


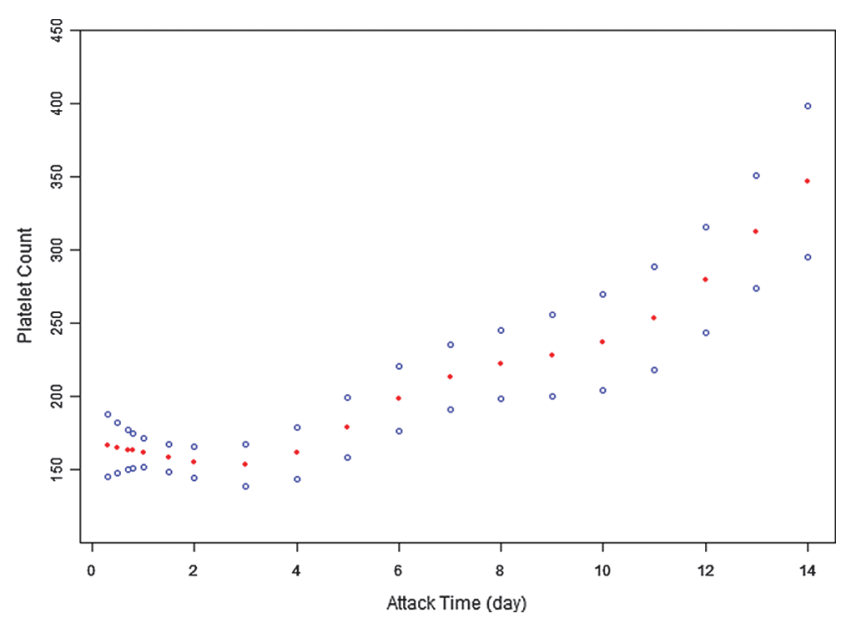

Fig. 1 A nonlinear relationship between attack time and admission platelet count in Chinese adults with Stanford type A acute aortic dissection after adjustment for age, sex, and smoking history.

with admission PLC with a $\mathrm{p}$ value $<0.10$ were selected. Then, these selected variables were used in multiple regression analyses to estimate the $\beta$ coefficient for admission PLC and their corresponding 95\% confidence intervals (CIs). Smoothing spline plots of independent factors related to admission PLC were created, and threshold effect analysis of independent factors on PLC was performed. $p$ values less than 0.05 were considered statistically significant.

\section{Results}

The flow diagram is present in Supplemental Fig. 1. (The supplementary material is available at ATCS online.) According to inclusion and exclusion criteria, a total of 130 patients undergoing Stanford type A AAD surgery were ultimately included in this study. The mean age of all of the patients was 46.8 years (range, 21-72 years), and the majority of patients were male (75.4\%). Baseline characteristics are presented in Table 1. Univariate analysis showed that the nine variables, including Age, Sex, BMI, History of smoking, Attack time, WBC, FDP, TFPI, and TXB2 were associated with admission PLC (Table 1). Multiple regression analyses of these variables showed that age $(\beta:-1.069,95 \% \mathrm{CI}:-2.109,-0.029, \mathrm{p}=0.0462)$, sex ( $\beta:-29.973,95 \% \mathrm{CI}:-56.512,-3.433 ; \mathrm{p}=0.0289)$, plasma levels of TFPI ( $\beta$ : 0.197, 95\% CI: 0.039, 0.354; $\mathrm{p}=0.0158$ ), plasma levels of FDP ( $\beta$ : $-0.476,95 \%$ CI: $-0.879,0.074$; $\mathrm{p}=0.0221)$, and attack time ( $\beta$ : $11.125,95 \%$ CI: 7.963 , 14.287; $\mathrm{p}<0.0001)$ were significantly associated with admission PLC with or without adjusting for factors
(Table 2). After adjusting for conventional clinical factors, a nonlinear relationship between attack time and admission PLC was observed (Fig. 1). The admission PLC increased with increasing attack time up to the 3 days (turning point) ( $\beta$ : 16.2, 95\% CI: 12.1, 20.2; $\mathrm{p}<0.001$ ) (Table 3).

\section{Discussion}

The present study showed what independent factors correlate with admission PLC in patients with Stanford type A AAD. Specifically, increasing age, male patients, patients with lower serum levels of TFPI and higher serum levels of FDP, and patients with a shorter attack time were significantly associated with lower PLC at admission. After adjusting for age, sex, and smoking history, a U-shaped association between attack time and PLC was observed and a longer attack time ( $\geq 3$ days) was associated with higher PLC.

A low PLC at admission could reflect a consumption process second to inflammation and thrombosis in AAD. Patients with a greater extent of dissection associated with a lower PLC and higher serum C-reactive protein (CRP) in AAD could account for this hypothesis. ${ }^{9)}$ Huang et al. ${ }^{12)}$ found that low admission PLC was significantly associated with higher in-hospital mortality in patients with type A AAD. Consistent with these results, in our previous study, ${ }^{16)}$ lower preoperative PLC was a risk factor for prolonged mechanical ventilation (PMV) after type A AAD surgery, which associated with increased in-hospital mortality and decreased 1-year survival rate compared with the non-PMV group.

More recently, it has been reported that PLC decreases during aging, and is higher in women than in men. ${ }^{17)}$ In The Moli-sani Study, 21,635 subjects were recruited and consistent associations with low PLC and higher risk for total mortality were found. ${ }^{18)}$ The Moli-sani Study evaluated the association of age- and sex-specific ranges of PLC with the risk of all-cause mortality in a large population-based epidemiologic cohort. In the present study, we found that the PLC in patients with AAD decreased $1.069\left(10^{9} / \mathrm{L}\right)$ with each 1-year increase in age, and female PLC exceeded those of men by 29.973 $\left(10^{9} / \mathrm{L}\right)$. The decrease in PLC in the elderly may reflect a reduction in hematopoietic stem cell reserve or in the quality of the individual stem cells that occurs during aging. ${ }^{19)}$ Another finding of our study concerns genderrelated differences in PLC in patients with AAD. The reduction of body iron in menstruating women is probably related to their higher PLC because platelet 
Table 1 Baseline characteristics of patients and univariate regression analysis for admission platelet count

\begin{tabular}{|c|c|c|}
\hline \multirow{2}{*}{ Variables } & \multirow{2}{*}{ Characteristics } & Univariate analysis \\
\hline & & $\beta(95 \% \mathrm{CI}) \mathrm{p}$ value \\
\hline Number (n) & 130 & \\
\hline Age (year) & $46.86 \pm 10.52$ & $-1.166(-2.287,-0.045) 0.0436$ \\
\hline \multicolumn{3}{|l|}{ Sex } \\
\hline Females, n (\%) & $32(24.615)$ & 1 \\
\hline Males, n (\%) & $98(75.38)$ & $-30.126(-57.347,-2.905) 0.0319$ \\
\hline $\operatorname{BMI}\left(\mathrm{kg} / \mathrm{m}^{2}\right)$ & $25.90 \pm 3.44$ & $0.567(-2.883,4.017) 0.7478$ \\
\hline History of smoking, $\mathrm{n}(\%)$ & $66(50.77)$ & $-20.098(-43.725,3.528) 0.0979$ \\
\hline History of hypertension, $\mathrm{n}(\%)$ & $94(72.31)$ & $-0.518(-27.200,26.163) 0.9697$ \\
\hline History of DM, n (\%) & $4(3.08)$ & $47.452(-21.194,116.099) 0.1778$ \\
\hline Attack time (d) & $2.00(1.00-5.00)$ & $11.229(8.266,14.193)<0.0001$ \\
\hline Preoperative SBP (mm Hg) & $113.24 \pm 18.24$ & $0.106(-0.555,0.766) 0.7537$ \\
\hline Preoperative DBP (mm Hg) & $56.02 \pm 11.89$ & $0.059(-0.954,1.073) 0.9090$ \\
\hline $\operatorname{LVEF}(\%)$ & $62.58 \pm 8.39$ & $-0.636(-2.070,0.798) 0.3863$ \\
\hline $\operatorname{LVEDD}(\mathrm{mm})$ & $51.40 \pm 7.47$ & $0.953(-0.685,2.591) 0.2564$ \\
\hline EuroSCORE & $5(5,6)$ & $-3.831(-14.966,7.304) 0.5013$ \\
\hline $3 \mathrm{n}(\%)$ & $19(14.62)$ & - \\
\hline $4 \mathrm{n}(\%)$ & $5(3.85)$ & - \\
\hline $5 \mathrm{n}(\%)$ & $69(53.08)$ & - \\
\hline $6 \mathrm{n}(\%)$ & $30(23.08)$ & - \\
\hline $7 \mathrm{n}(\%)$ & $5(3.85)$ & - \\
\hline $8 \mathrm{n}(\%)$ & $2(1.54)$ & - \\
\hline \multicolumn{3}{|l|}{ Aortic regurgitation $(\mathrm{n}=129)$} \\
\hline Non-regurgitation $\mathrm{n}(\%)^{*}$ & $27(20.93)$ & 1 \\
\hline Mild-regurgitation n (\%) & $52(40.31)$ & $18.137(-14.242,50.517) 0.2744$ \\
\hline Moderate-regurgitation n (\%) & $22(17.05)$ & $4.008(-35.197,43.213) 0.8415$ \\
\hline Severe-regurgitation $\mathrm{n}(\%)$ & $28(21.71)$ & $5.930(-30.888,42.748) 0.7528$ \\
\hline \multicolumn{3}{|l|}{ Serum variables } \\
\hline $\mathrm{Hb}(\mathrm{g} / \mathrm{L})$ & $12.80(11.90-13.50)$ & $0.342(-0.748,1.432) 0.5399$ \\
\hline WBC $\left(10^{9} / \mathrm{L}\right)$ & $9.44(7.54-10.82)$ & $-3.319(-6.834,0.197) 0.0666$ \\
\hline $\mathrm{LAC}(\mathrm{mmol} / \mathrm{L})$ & $1.00(0.80-1.40)$ & $-8.312(-20.265,3.642) 0.1753$ \\
\hline \multicolumn{3}{|l|}{ Coagulation/fibrinolysis } \\
\hline FIB $(g / L)$ & $3.78(2.85-4.62)$ & $4.718(-3.982,13.418) 0.2899$ \\
\hline $\mathrm{FDP}(\mathrm{mg} / \mathrm{L})$ & $10.20(6.20-18.70)$ & $-0.831(-1.305,-0.358) 0.0008$ \\
\hline PAI-1 (ng/mL) & $0.71 \pm 0.19$ & $-5.119(-67.795,57.557) 0.8731$ \\
\hline $\mathrm{TF}(\mathrm{ng} / \mathrm{mL})$ & $3.48(2.62-4.88)$ & $0.293(-4.546,5.133) 0.9056$ \\
\hline TFPI (ng/mL) & $146.42 \pm 65.20$ & $0.257(0.079,0.436) 0.0054$ \\
\hline \multicolumn{3}{|l|}{ Inflammatory } \\
\hline IL-6 (pg/mL) & $56.19(46.47-70.50)$ & $0.170(-0.353,0.693) 0.5247$ \\
\hline IL-10 (pg/mL) & $78.38(43.68-114.85)$ & $0.176(-0.029,0.380) 0.0946$ \\
\hline HLE (ng/mL) & $2.23(1.67-2.63)$ & $3.457(-6.048,12.961) 0.4772$ \\
\hline $\mathrm{TNF} \alpha(\mathrm{pg} / \mathrm{mL})$ & $53.77(38.61-70.78)$ & $0.091(-0.295,0.478) 0.6444$ \\
\hline \multicolumn{3}{|l|}{ ROS } \\
\hline $\operatorname{MDA}(\mathrm{nmol} / \mathrm{mL})$ & $3.30 \pm 0.59$ & $-12.927(-33.006,7.151) 0.2093$ \\
\hline MPO (units/L) & $46.93 \pm 15.01$ & $0.541(-0.269,1.350) 0.1932$ \\
\hline TAOC (units/mL) & $7.17 \pm 1.87$ & $.713(-2.659,10.085) 0.2555$ \\
\hline TSOD (units/mL) & $109.15 \pm 42.86$ & $0.058(-0.231,0.347) 0.6964$ \\
\hline \multicolumn{3}{|l|}{ Platelet and endothelial cell } \\
\hline Platelet count $\left(10^{9} / \mathrm{L}\right)$ & $168.77(136.25-204.00)$ & - \\
\hline VEGF (pg/mL) & $198.92(135.86-338.26)$ & $-0.012(-0.068,0.044) 0.6757$ \\
\hline $\mathrm{PGI}_{2}(\mathrm{pg} / \mathrm{mL})$ & $49.69(25.19-103.20)$ & $0.026(-0.098,0.150) 0.6807$ \\
\hline $\mathrm{TXB}_{2}(\mathrm{pg} / \mathrm{mL})$ & $154.82 \pm 98.94$ & $-0.120(-0.240,-0.001) 0.0502$ \\
\hline
\end{tabular}

Data are given as numbers, percentage, mean \pm standard deviation or median IQR. *: Non-regurgitation group is the reference group. \#: EuroSCORE is defined as continuous variable. BMI: Body Mass Index; CI: confidence interval; DM: diabetes mellitus; DBP: upper limb diastolic blood pressure; EuroSCORE: European system for cardiac operative risk evaluation; FDP: fibrinogen degradation product; FIB: fibrinogen; Hb: hemoglobin; HLE: human leukocyte elastase; IL: interleukin; LAC: lactic acid; LVEF: left ventricular ejection fraction; LVEDD: left ventricular end diastolic diameter; MDA: methane dicarboxylic aldehyde; MPO: myeloperoxidase; $\mathrm{PGI}_{2}$ : prostaglandin $\mathrm{I}_{2}$; PLC: platelet count; ROS: reactive oxygen species; SBP: upper limb systolic blood pressure; TAOC: total anti-oxidation capacity; TSOD: total superoxide dismutase; TF: tissue factor; TFPI: tissue factor pathway inhibitor; TNF $\alpha$ : tumor necrosis factor $\alpha$; $\mathrm{TXB}_{2}$ : thromboxane $\mathrm{B}_{2}$; VEGF: vascular endothelial growth factor; WBC: white blood cells; IQR: interquartile range; PAI-1: plasminogen activator inhibitor-1 
Table 2 Multivariate regression analysis for admission platelet count with or without adjustment $(\mathbf{n}=124)$

\begin{tabular}{lccc}
\hline \multirow{2}{*}{ Exposure } & Crude model & Multivariate-adjusted model 1 & Multivariate-adjusted model 2 \\
\cline { 2 - 4 } & $\beta(95 \% \mathrm{CI}) \mathrm{p}$ value & $\beta(95 \% \mathrm{CI}) \mathrm{p}$ value & $\beta(95 \% \mathrm{CI}) \mathrm{p}$ value \\
\hline Sex & 1 & 1 & 1 \\
$\quad$ Females* & & $-30.19(-56.603,-3.778) 0.0270$ & $-29.973(-56.512,-3.433) 0.0289$ \\
$\quad$ Males & $-31.579(-54.433,-8.724) 0.0078$ & $-1.162(-2.160,-0.164) 0.0243$ & $-1.069(-2.109,-0.029) 0.0462$ \\
Age (per 1 year) & $-1.181(-2.159,-0.203) 0.0196$ & $0.228(0.083,0.373) 0.0025$ & $0.197(0.039,0.354) 0.0158$ \\
TFPI (per 1 ng/mL) & $0.229(0.085,0.373) 0.0023$ & $-0.476(-0.876,-0.077) 0.0212$ & $-0.476(-0.879,-0.074) 0.0221$ \\
FDP (per 1 mg/L) & $-0.476(-0.874,-0.078) 0.0208$ & $10.493(7.616,13.37)<0.0001$ & $11.125(7.963,14.287)<0.0001$ \\
Attack time (per 1 day) & $10.492(7.626,13.357)<0.0001$ & 10.45 \\
\hline
\end{tabular}

Crude: no adjustment. Model I: adjusted for history of smoking. Model II: adjusted for history of smoking, TXB ${ }_{2}$, IL-10, and WBC. *: Females group is the reference group. CI: confidence interval; TFPI: tissue factor pathway inhibitor; FDP: fibrinogen degradation product; $\mathrm{TXB}_{2}$ : thromboxane $\mathrm{B}_{2}$; IL: interleukin; WBC: white blood cells

Table 3 Threshold effect analysis of attack time and platelet count in Chinese adults with Stanford type $A$ acute aortic dissection after adjustment for age, sex, and smoking history

\begin{tabular}{lc}
\hline Exposure: attack time (day) & Platelet count $\left(10^{9} / \mathrm{L}\right)^{\mathrm{a}}$ \\
\hline Model I & $10.9(8.0,13.8)<0.001$ \\
$\quad$ One line slope & 3 \\
Model II & $-8.8(-20.2,2.7) 0.136$ \\
Turning point $(\mathrm{K})$ & $16.2(12.1,20.2)<0.001$ \\
$\quad$ < K slope 1 & $24.9(10.8,39.0) 0.001$ \\
> K slope 2 & $144.0(125.6,162.5)$ \\
Slope 2-Slope 1 & 0.001 \\
Predicted at K & $1.4875,11$ \\
LRT test & \\
95\% CI of K &
\end{tabular}

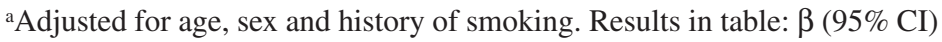

p value. LRT test: likelihood-ratio test; CI: confidence interval

production is stimulated by moderate iron deficiency. ${ }^{20,21)}$ However, hormonal differences between men and women could also be involved, as estrogens were shown to favor platelet formation in vitro and in vivo. ${ }^{22)}$ During the progress of $\mathrm{AAD}$, there is a significant correlation between inflammation and platelet activation, which may be induced by a tear in the aortic wall and exposed collagen. ${ }^{9,23)}$ Therefore, there was a more profound effect on age- and sex-specific PLC in patients with AAD.

In this study, TFPI level was positively correlated with PLC, but FDP level was negatively correlation with PLC, indicating that PLC was modulated by active coagulation and fibrinolytic systems. During the progress of AAD, blood flow through the nonendothelialized false lumen and turbulence formation is a powerful activator of the coagulation and fibrinolytic systems. ${ }^{24)}$ The hemostatic system is activated, resulting in clotting factor consumption and ultimately coagulopathy, leading to intense activation of fibrinolysis by fibrin formation and degra- dation. TFPI regulates the extrinsic pathway of blood coagulation to inhibit either $\mathrm{TF}^{25}$ ) or early forms of prothrombinase. It is also essential for the dampening of thrombin-mediated platelet activation, ${ }^{26)}$ which decreases platelet consumption to elevate PLC. FDP is the degradation product of fibrous protein by plasmin, which participates in the development and progression of atherosclerosis and thrombus, and is positively correlated with the possibility of thrombosis type AAD. ${ }^{27)}$ In 1991 , Winters et al. ${ }^{28)}$ found that plasmin-derived FDPs could promote platelet aggregation in response to activation with various agonists, which increased platelet consumption to reduce PLC.

The current study shows that in the interval between dissection onset and surgery, there is a time-dependent effect on PLC, which revealed that admission PLC increased $16.2\left(10^{9} / \mathrm{L}\right)$ by a $1-\mathrm{d}$ increase in attack time up to 3 days. Thus, there is a reasonable prospect of getting the pathophysiology changes as the progress of AAD. $\mathrm{Xu}$ et al. ${ }^{29)}$ reported that there was evidence of adventitial 
inflammation, such as neutrophils peaking at $24 \mathrm{~h}$, mitotic figures at $28 \mathrm{~h}$, apoptotic bodies at $60 \mathrm{~h}$, and eosinophils at $23 \mathrm{~h}$ in 40 aortas. Albini et al. ${ }^{30)}$ also reported that D-dimer levels significantly increased after dissection onset and then dropped after 2 days, but still remained elevated over a $10-\mathrm{d}$ period in $\mathrm{AAD}$ patients.

\section{Study Limitations}

This study had several limitations. First, it was conducted at only one university center, and the number of cases was relatively small for a relatively short period time. Second, the time of the initiating event was chosen by necessity as the onset of pain. It is quite possible that the initiation of the intimal tear may predate that of the dissection itself with pain or painless. Thus, the attack time does not accurately reflect the time between dissection onset and surgery. Third, all of the patients in our study were suitable for emergency AAD surgery without severe malperfusion and unstable hemodynamics, which are not representative of all patients. Forth, Stanford type A AAD demonstrated the similar volume of aortic dissection; however, we were not able to obtain highly detailed information to evaluate the volume of false lumen and thrombogenesis. Finally, it is not yet clear that there is any true cause-and-effect relationship between independent factors and PLC for the crosssectional study. Therefore, a large cohort study is recommended to address these problems.

\section{Conclusion}

The present study found that increasing age, male patients, patients with lower serum levels of TFPI and higher serum levels of FDP, and patients with a shorter attack time were significantly associated with lower PLC at admission. Moreover, the turning point of attack time is 3 days after the onset of dissection.

\section{Ethics Approval and Consent to Participate}

This study was in agreement with the guidelines of the Ethics Committee of the Beijing Anzhen hospital. All patients gave verbal and written informed consent prior to enrollment.

\section{Contributors}

MJ was responsible for the original idea, which was co-developed by all authors. All authors (SWL, JKL,
WPC, JMZ, and MJ) developed the conception and design of this manuscript. JKL, WPC, and JMZ validated the provision of study materials or patients and SWL carried out all the data management and statistical analyses, which was supervised by MJ. SWL and MJ were responsible for writing the manuscript, which was critically revised by all coauthors.

\section{Funding}

The study was supported by grants from Beijing Municipal Science \& Technology Commission (No. Z151100004015133, No. Z161100000513067, and No. Z171100001017083), and National Science and Technology support program of China (No.2015BAI12B03). The funding institution had no influence on the design, analysis, and publication of this study.

\section{Data Sharing Statement}

The dataset used and analyzed during the current study are available from the corresponding author upon reasonable request.

\section{Acknowledgment}

We thank LetPub (www.letpub.com) for its linguistic assistance during the preparation of this manuscript.

\section{Disclosure Statement}

All authors have no conflict of interest.

\section{References}

1) Sheikh AS, Ali K, Mazhar S. Acute aortic syndrome. Circulation 2013; 128: 1122-7.

2) Golledge J, Eagle KA. Acute aortic dissection. Lancet 2008; 372:55-66.

3) Qiu Z, Chen L, Cao H, et al. Mechanical strain induced expression of matrix metalloproteinase-9 via stretch-activated channels in rat abdominal aortic dissection. Med Sci Monit 2017; 23: 1268-76.

4) Luo J, Fu X, Zhou Y, et al. Aortic remodeling following sun's procedure for acute type A aortic dissection. Med Sci Monit 2017; 23: 2143-50.

5) Hagan PG, Nienaber CA, Isselbacher EM, et al. The international registry of acute aortic dissection (IRAD): new insights into an old disease. JAMA 2000; 283: 897-903.

6) Tanaka M, Kawahito K, Adachi H, et al. Platelet dysfunction in acute type A aortic dissection evaluated by 
the laser light-scattering method. J Thorac Cardiovasc Surg 2003; 126: 837-41.

7) Sbarouni E, Georgiadou P, Analitis A, et al. Significant changes in platelet count, volume and size in acute aortic dissection. Int J Cardiol 2013; 168: 4349-50.

8) Vieira-de-Abreu A, Campbell RA, Weyrich AS, et al. Platelets: versatile effector cells in hemostasis, inflammation, and the immune continuum. Semin Immunopathol 2012; 34: 5-30.

9) Zhang S, Qian H, Yang Q, et al. Relationship between the extent of dissection and platelet activation in acute aortic dissection. J Cardiothorac Surg 2015; 10: 162 .

10) Qin C, Zhang H, Gu J, et al. Dynamic monitoring of platelet activation and its role in post-dissection inflammation in a canine model of acute type A aortic dissection. J Cardiothorac Surg 2016; 11: 86.

11) Li DZ, Chen QJ, Sun HP, et al. Mean platelet volume to platelet count ratio predicts in-hospital complications and long-term mortality in type A acute aortic dissection. Blood Coagul Fibrinolysis 2016; 27: 653-9.

12) Huang B, Tian L, Fan X, et al. Low admission platelet counts predicts increased risk of in-hospital mortality in patients with type A acute aortic dissection. Int J Cardiol 2014; 172: e484-6.

13) Cheng Y, Jin M, Dong X, et al. Mechanism and early intervention research on ALI during emergence surgery of Stanford type-A AAD: study protocol for a prospective, double-blind, clinical trial. Medicine (Baltimore) 2016; 95: e5164.

14) Yan TD, Tian DH, Lemaire SA, et al. Standardizing clinical end points in aortic arch surgery: a consensus statement from the International Aortic Arch Surgery Study Group. Circulation 2014; 129:1610-6.

15) Kimura $N$, Ohnuma $T$, Itoh $S$, et al. Utility of the Penn classification in predicting outcomes of surgery for acute type A aortic dissection. Am J Cardiol 2014; 113: 724-30.

16) Jin M, Ma WG, Liu S, et al. Predictors of prolonged mechanical ventilation in adults after acute type-A aortic dissection repair. J Cardiothorac Vasc Anesth 2017; 31: 1580-7.

17) Biino G, Santimone I, Minelli C, et al. Age- and sex-related variations in platelet count in Italy: a proposal of reference ranges based on 40987 subjects' data. PLoS ONE 2013; 8: e54289.
18) Bonaccio M, Di Castelnuovo A, Costanzo S, et al. Age-sex-specific ranges of platelet count and all-cause mortality: prospective findings from the MOLI-SANI study. Blood 2016; 127: 1614-6.

19) Warren LA, Rossi DJ. Stem cells and aging in the hematopoietic system. Mech Ageing Dev 2009; 130: 46-53.

20) Park MJ, Park PW, Seo YH, et al. The relationship between iron parameters and platelet parameters in women with iron deficiency anemia and thrombocytosis. Platelets 2013; 24: 348-51.

21) Kadikoylu G, Yavasoglu I, Bolaman Z, et al. Platelet parameters in women with iron deficiency anemia. J Natl Med Assoc 2006; 98: 398-402.

22) Nagata Y, Yoshikawa J, Hashimoto A, et al. Proplatelet formation of megakaryocytes is triggered by autocrine-synthesized estradiol. Genes Dev 2003; 17: 2864-9.

23) Stevic I, Chan HH, Chan AK. Carotid artery dissections: thrombosis of the false lumen. Thromb Res 2011; 128: 317-24.

24) Guan XL, Wang XL, Liu YY, et al. Changes in the hemostatic system of patients with acute aortic dissection undergoing aortic arch surgery. Ann Thorac Surg 2016; 101: 945-51.

25) Maroney SA, Mast AE. Platelet tissue factor pathway inhibitor modulates intravascular coagulation. Thromb Res 2012; 129: S21-2.

26) Ellery PE, Maroney SA, Cooley BC, et al. A balance between TFPI and thrombin-mediated platelet activation is required for murine embryonic development. Blood 2015; 125: 4078-84.

27) Hagiwara A, Shimbo T, Kimira A, et al. Using fibrin degradation products level to facilitate diagnostic evaluation of potential acute aortic dissection. J Thromb Thrombolysis 2013; 35: 15-22.

28) Winters KJ, Santoro SA, Miletich JP, et al. Relative importance of thrombin compared with plasmin-mediated platelet activation in response to plasminogen activation with streptokinase. Circulation 1991; 84: 1552-60.

29) $\mathrm{Xu} \mathrm{L}$, Burke A. Acute medial dissection of the ascending aorta: evolution of reactive histologic changes. Am J Surg Pathol 2013; 37: 1275-82.

30) Albini P, Barshes NR, Russell L, et al. D-dimer levels remain elevated in acute aortic dissection after $24 \mathrm{~h}$. J Surg Res 2014; 191: 58-63. 